\title{
Estimation of the signal component from random equivalent and non-coherent sampling measurements
}

\author{
Dušan Agrež \\ University of Ljubljana, Faculty of Electrical Engineering, Trzaska 25, 1000 Ljubljana, Slovenia
}

\begin{abstract}
Estimations of the signal component parameters in the case of random equivalent time sampling and under non-coherent sampling condition comprise two main error contributions: spectral leakage effect due to non-coherency and additional noise due to the randomization of sampling intervals. In the estimation procedure the non-parametric interpolated DFT approach has to be used first to estimate the component frequency and, after that, an iterative 4-parametric sine-fit algorithm should be used for other component parameters (amplitude and phase). Their estimations are possible when the duty ratio of random samples from the total samples in the non-coherent measurement interval is above 0.1 . With these duty ratios of random samples it is possible to achieve error levels of 0.001 bins of the frequency estimations in relation to the estimation on full ensemble of points.
\end{abstract}

\section{Section: RESEARCH PAPER}

Keywords: random equivalent time sampling; non-coherent condition; signal component; interpolated DFT; parametric estimation

Citation: Dušan Agrež, Estimation of signal component from random equivalent and non-coherent sampling measurements, Acta IMEKO, vol. 6, no. 4, article 8, December 2017, identifier: IMEKO-ACTA-06 (2017)-04-08

Section Editor: Alexandru Salceanu, Technical University of lasi, Romania

Received May 5, 2017; In final form June 27, 2017; Published December, 2017

Copyright: @ 2017 IMEKO. This is an open-access article distributed under the terms of the Creative Commons Attribution 3.0 License, which permits unrestricted use, distribution, and reproduction in any medium, provided the original author and source are credited

Corresponding author: Dušan Agrež, e-mail: dusan.agrez@fe.uni-lj.si

\section{INTRODUCTION}

High-speed digitizing of waveforms plays an important part in broadband measurements and one of the possibilities to increase the sampling frequency is the equivalent time sampling with reconstruction of the signal in the prolongation of the acquisition time [1]. In many applications of practical interest, we often wish to estimate signal parameters and reconstruct a signal from incomplete time samples, which can be attained non-uniformly and randomly as in the case of random equivalent sampling [2], [3]. Unlike the uniform sampling case, where the aliases are simply periodic replicas of the signal's original spectrum when the sampling theorem is fulfilled, random sampling theory shows that sparsing and randomization of the sampling intervals shape the aliases into a noise floor in the sampled spectrum [4]. By sampling signals non-uniformly, the average sample rate can be more than a magnitude lower than the Nyquist rate, provided that these signals have a relatively low information content as measured by the sparsity of their spectrum.

Random equivalent time sampling is a technique which is used for achieving high sample rates with reduction of the equivalent sampling interval $t_{\mathrm{S} \text {,eq }}$. It is applicable only for periodic signals. It is usually used in digital oscilloscopes and some other high-speed sampling applications [5], [6]. The general idea behind equivalent time sampling is shown in Figures 1 to 6 . Figure 1 shows one sinusoidal signal component $g$, which can be generally expressed as $w\left(n t_{\mathrm{S}, \mathrm{eq}}\right) \cdot \sum_{m=0}^{M-1} A_{m} \sin \left(2 \pi f_{m} n t_{\mathrm{S}, \mathrm{eq}}+\varphi_{m}\right)\left(f_{m}, A_{m}\right.$, and $\varphi_{m}$ are frequency, amplitude, and phase of the particular component). The signal is presented by randomly sampled points with low 'duty ratio' $D_{\mathrm{rs}}=N_{\mathrm{rs}} / N$, where $N_{\mathrm{rs}}$ is the number of random samples from a total of $N$ samples in the non-coherent measurement interval $T_{\mathrm{M}}=N t_{\mathrm{S}, \mathrm{eq}}$. The basic raster of $N$ samples is equidistant with the sampling interval $t_{\mathrm{S} \text {,eq }}$ [7]. The whole acquired signal contains 3.8 cycles of the sine $\theta_{m}=f_{m} / \Delta f=f_{m} \cdot N t_{\mathrm{S} \text { eq }} \doteq 3.8$ on $N=512$ points and samples are captured randomly to the triggering point (signal 


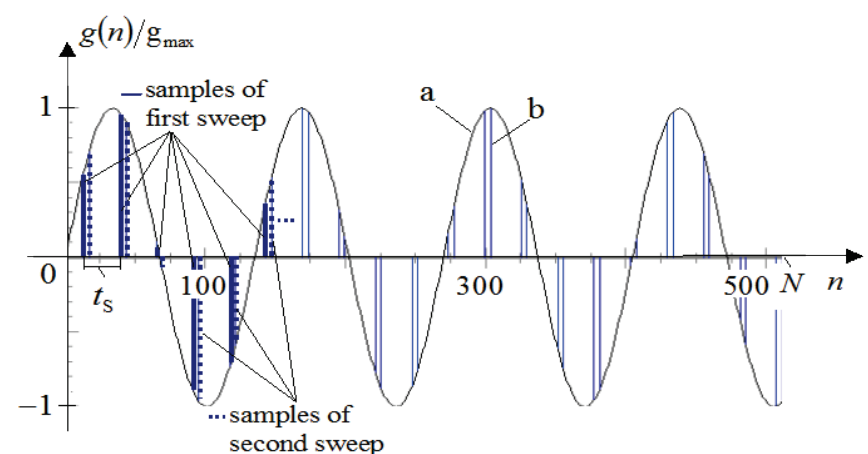

Figure 1. Signals of random sampling: a-original signal, b-random sampled signal; $N=512, \theta_{\mathrm{S}}=19.7, \theta_{m}=3.8, A=1, \varphi=0 \mathrm{deg}, D_{\mathrm{rs}} \approx 0.07$.

zero crossing with phase $0 \mathrm{deg}$ ) in two sweeps with fix sampling raster $t_{\mathrm{S}}=26 t_{\mathrm{S}, \mathrm{eq}}$. In Figure 1 the samples of the first sweep are represented by solid lines and the samples of the second sweep by dotted lines). In this figure the sampling theorem is fulfilled with a relative sampling frequency of $\theta_{\mathrm{S}}=f_{\mathrm{S}} / \Delta f=N t_{\mathrm{S} \text { eq }} / t_{\mathrm{S}}=512 / 26 \doteq 19.7>2 \cdot \theta_{\mathrm{m}}$.

Although the samples are randomly captured (essentially the first sample in one sweep) the systematic relation between the signal frequency and sampling frequency is clearly visible in the spectrum of the equivalent sampled signal (Figure 2 - line b) where we have well-known replicas of signal components with frequency $\theta_{m}$ around integer values of the sampling frequency $\theta^{\prime}=k \theta_{\mathrm{S}} \pm \theta_{m}(k=1,2, \ldots)$. With increasing the number of sweeps the duty ratio approaches $D_{\mathrm{rs}} \rightarrow 1$ and the component rises from the noise floor and replicas diminish (Figure 2 - line a).

If the Analog-to-Digital conversion is relatively slow compared to the signal period, the sampling raster relatively increases and the sampling theorem could not be fulfilled (see Figure 3:

$\left.t_{\mathrm{S}}=85 t_{\mathrm{S} \text { eq }} \rightarrow \theta_{\mathrm{S}}=N t_{\mathrm{S} \text {,eq }} / t_{\mathrm{S}}=512 / 85 \doteq 6.0<2 \cdot \theta_{\mathrm{m}}\right)$.

To fill the empty points by signal values more sweeps are needed for the same duty ratio (in Figure 3: six sweeps are needed for $D_{\mathrm{rs}} \approx 0.07$ ) but the systematic relation between sampling frequency and component frequency is still recognized (Figure 4 - line b).

If one reduces the number of samples of one sweep to 1 , what is the limit case, we get 'pure' random sampling (Figure 5: $\left.t_{\mathrm{S}}=512 t_{\mathrm{S}, \mathrm{eq}} \rightarrow \theta_{\mathrm{S}}=512 / 512=1<<2 \cdot \theta_{\mathrm{m}}\right)$.

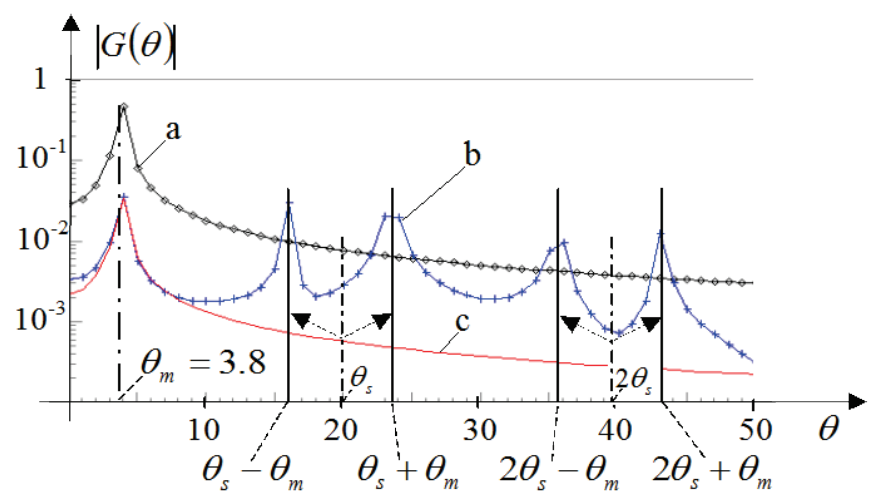

Figure 2. Spectra of signals from Figure 1: a - original signal, $\mathrm{b}$ - random sampled signal, c-reconstructed signal; $\theta_{\mathrm{S}}=19.7, \theta_{m}=3.8, D_{\mathrm{rs}} \approx 0.07$.

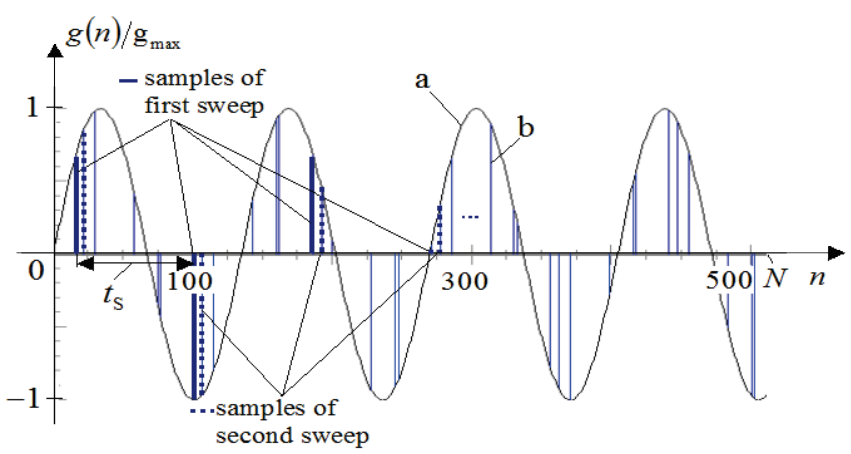

Figure 3. Signals of random sampling: a - original signal, $\mathrm{b}$ - random sampled signal; $N=512, \theta_{\mathrm{S}} \doteq 6.0, \theta_{m}=3.8, A=1, \varphi=0 \mathrm{deg}, D_{\mathrm{rs}} \approx 0.07$.

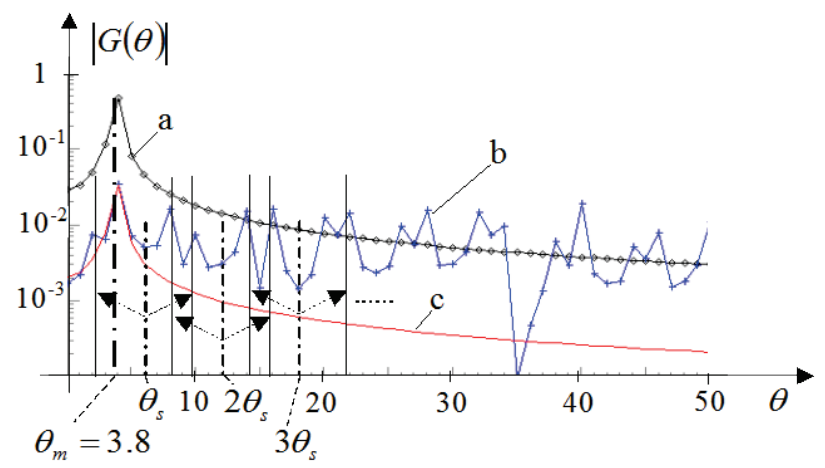

Figure 4. Spectra of signals from Figure 3: a - original signal, b - random sampled signal, c - reconstructed signal; $\theta_{\mathrm{S}} \doteq 6.0, \theta_{m}=3.8, D_{\mathrm{rs}} \approx 0.07$.

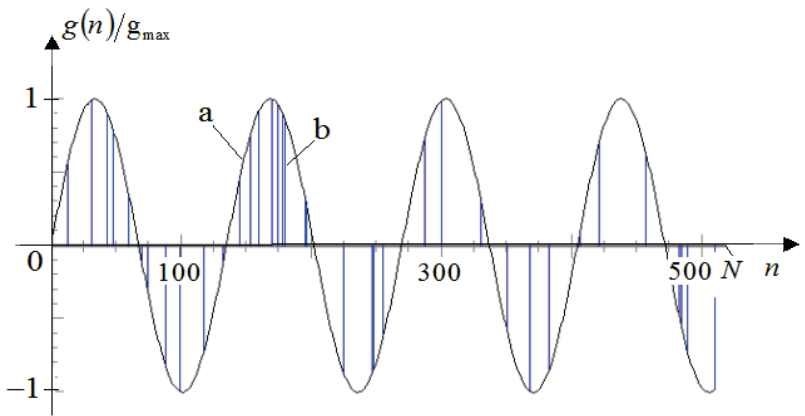

Figure 5. Signals of random sampling: $\mathrm{a}$ - original signal, $\mathrm{b}$ - random sampled signal; $N=512, \theta_{\mathrm{S}}=1, \theta_{m}=3.8, A=1, \varphi=0 \mathrm{deg}, D_{\mathrm{rs}} \approx 0.07$.

Due to relative sampling frequency of $\theta_{\mathrm{S}}=1$ all amplitude DFT coefficients are contaminated by a systematic sampling effect $\theta^{\prime}=k \pm \theta_{m}(k=1,2, \ldots)$ and beside noise of the acquisition signal we have also the 'noise' of random sampling (Figure 6 - line b). This fact has to be considered in estimations of the signal components.

Estimations of the component signal parameters are distorted due to the missing samples in the time domain [8] or due to large random sampling noise in the frequency domain (Figure 6 - line b) where SNR for the investigated component is very low when $D_{\mathrm{rs}}$ is low. Considering also the non-coherent sampling condition with leakage effect, where the measurement interval $T_{\mathrm{M}}$ is not an integer multiple of the signal period $\theta_{m}=T_{\mathrm{M}} / T_{m}=i_{m}+\delta_{m}$, parameter estimations are not an easy task [9], [10]. The leakage signal energy additionally spreads 


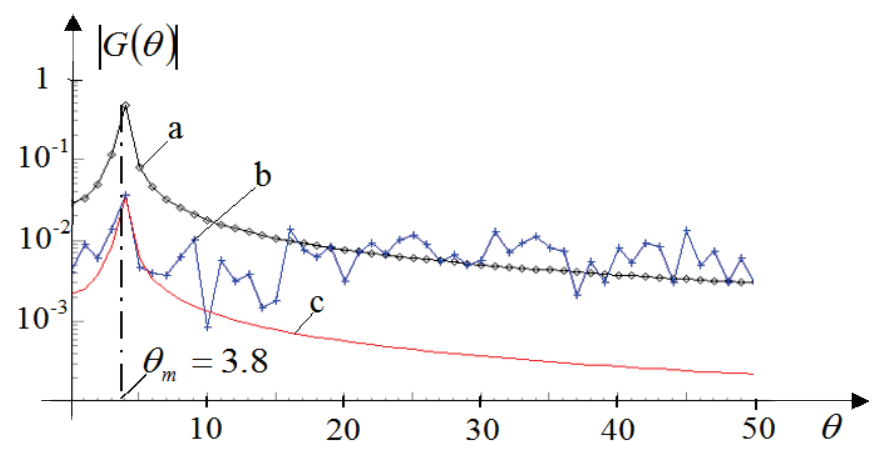

Figure 6. Spectra of signals from Figure 5: a - original signal, $\mathrm{b}$ - random sampled signal, c - reconstructed signal; $\theta_{\mathrm{S}}=1, \theta_{m}=3.8, D_{\mathrm{rs}} \approx 0.07$.

along the frequency axis and disturbs the DFT coefficients (Figure 6) [11], which cannot be removed as in the case of compressive sensing with the coherent sampling model [12], [13].

\section{ANALYSIS FOR PARAMETERS' ESTIMATIONS}

Accurate measurement of a multi-sine waveform is a classic problem in spectral analysis. It is well known that nonparametric algorithms based on the discrete Fourier transform (DFT) have to contend with spectral leakage, which affects both amplitude estimation accuracy and frequency resolution [14]. These methods usually estimate the signal component frequency as key parameter by searching for the maximum in the spectrum by using DFT [15]. Advanced windowed functions and interpolation schemes have been applied to compensate for the effects of leakage caused by incoherent sampling and finite frequency resolution problems [16], respectively. It is also common knowledge that approaches based on a parametric signal model can achieve much better frequency resolution, but this is obtained at the price of greater complexity [17].

For estimations of the signal parameters from sparse sampled periodic signals two methods are investigated and compared: the non-parametric interpolated DFT and the sinefit parametric approach [18]. In the first approach, one can choose the number of DFT coefficients in interpolation and the window function $\mathrm{w}\left({ }^{*}\right)$ [16]. In the second approach, a number of parameters has to be defined: non-iterative 3parametric sine-fit estimation when the component frequency is known or 4-parametric estimation when also the frequency has to be estimated in the iterative procedure [17].

In the first approach typically two or three largest DFT coefficients $G\left(i_{m}-1\right), G\left(i_{m}\right), G\left(i_{m}+1\right)$ and Rife-Vincent windows class I with suitable order $P$ are used [16]:

- Two-point frequency estimation where $s$ is a sign of displacement $\delta_{m}$ :

$\theta_{m}=i_{m}+{ }_{2} \delta_{m} \doteq i_{m}+s \frac{(P+1) \cdot\left|G\left(i_{m}+s\right)\right|-P \cdot\left|G\left(i_{m}\right)\right|}{\left|G\left(i_{m}\right)\right|+\left|G\left(i_{m}+s\right)\right|}$

- Three-point frequency estimation:

$\theta_{m}=i_{m}+{ }_{3} \delta_{m} \doteq i_{m}+(P+1) \cdot \frac{\left|G\left(i_{m}+1\right)\right|-\left|G\left(i_{m}-1\right)\right|}{\left|G\left(i_{m}-1\right)\right|+2\left|G\left(i_{m}\right)\right|+\left|G\left(i_{m}+1\right)\right|}$

- One-point amplitude estimation:

$$
{ }_{1} A_{m} \doteq 2\left|\frac{2^{2 P}}{(2 P) !} \cdot \frac{\pi \delta_{m}}{\sin \left(\pi \delta_{m}\right)} \cdot \prod_{l=1}^{P}\left(l^{2}-\delta_{m}^{2}\right)\right|\left|G\left(i_{m}\right)\right|
$$

- Three-point amplitude estimation:

$$
\begin{aligned}
&{ }_{3} A_{m} \doteq 2\left|\frac{2^{2 P}}{(2 P+2) !} \cdot \frac{\pi \delta_{m}}{\sin \left(\pi \delta_{m}\right)} \cdot \prod_{l=1}^{P+1}\left(l^{2}-\delta_{m}^{2}\right)\right| \\
& \cdot\left(\left|G\left(i_{m}-1\right)\right|+2\left|G\left(i_{m}\right)\right|+\left|G\left(i_{m}+1\right)\right|\right)
\end{aligned}
$$

- Three-point phase estimation with the arguments of the three largest local DFT coefficients $\varphi_{i_{m}}=\arg \left[G\left(i_{m}\right)\right]$ :

$$
{ }_{3} \varphi_{m}=\frac{\left(1-\delta_{m}\right) \varphi_{i_{m}-1}+4 \varphi_{i_{m}}+\left(1+\delta_{m}\right) \varphi_{i_{m}+1}}{6}-\frac{2 a \delta_{m}}{3}+\frac{\pi}{2}
$$

Since the sparse sampling considerably increases the noise in the frequency domain (Figure 6) it is not suitable to increase the number of interpolation DFT coefficients in interpolations due to their distortions. Figure 7 shows the mean values of the absolute relative errors of the amplitude DFT coefficients ( $e\left|G_{\mathrm{rs}}\left(i_{m}\right)\right|, e\left|G_{\mathrm{rs}}\left(i_{m}+1\right)\right|$, and $\left.e\left|G_{\mathrm{rs}}\left(i_{m}-1\right)\right|\right)$ around the local peak in relation to the original spectrum on $N=512$ samples normalized by the duty ratio $|G(i)|^{*}=|G(i)| \cdot D_{\mathrm{rs}}$ in (6) (see Figures 2,4,6 - line c).

$e\left(\left|G_{\mathrm{rs}}(i)\right|\right)=\frac{\left|G_{\mathrm{rs}}(i)\right|-|G(i)|^{*}}{|G(i)|^{*}}$

Analyses of Figure 7 show that it is better to use two-point estimation for frequency to determine the position of the measurement component $\delta_{m}=\theta_{m}-i_{m}$ between DFT
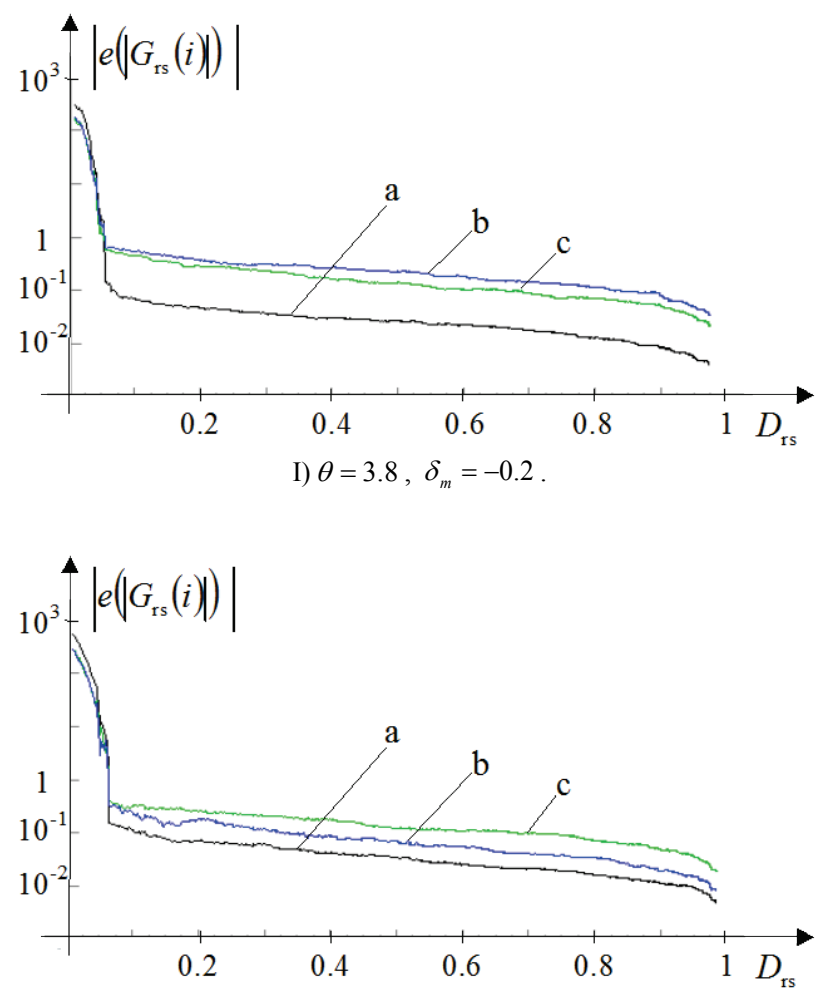

II) $\theta=4.4, \delta_{m}=0.4$.

Figure 7. Mean values of the absolute relative errors of the amplitude DFT coefficients; a $-e\left|G_{\mathrm{rs}}(i)\right|, \mathrm{b}-e\left|G_{\mathrm{rs}}(i+1)\right|, \mathrm{c}-e\left|G_{\mathrm{rs}}(i-1)\right|, A=1, \varphi=0 \mathrm{deg}$, $N_{\text {repeat }}=100$ trials. 
coefficients $G\left(i_{m}\right)$ and $G\left(i_{m}+1\right)$ surrounding the component $m$ as the key parameter, due specific distortion in sparse sampling. This conclusion can be supported also by analyzing the error characteristics using two and three- point estimations (Figure 8) [16].

Figure 8 shows collected minimal curves of the maximal bias errors of the frequency estimations due to phase changing with the two-point (IDFT-2p) and three-point (IDFT-3p) interpolations of the DFT using RV-I windows and the corresponding orders $P=1 \ldots 6$ with which these minimal values have been obtained. It can be seen that bias errors are on the same level with two (1) or three-point (2) estimations but window order $P$ has to be increased with two-point estimation.

Whenever there is no information of frequency content of the signal, the component frequency estimation is the first step and the key to estimate other parameters (amplitude, phase, and sometimes also damping). This is valid for both the parametric and the non-parametric approach. Results in Figures 9 and 10 confirm above presumptions, where the frequency estimation error is defined as $|E(\theta)|=\left|\theta_{\text {est. }}-\theta_{\text {true }}\right|$.

It can be noticed that the relative number of random samples $D_{\mathrm{rs}}=N_{\mathrm{rs}} / N$ has to increase at least to 0.1 when the frequency estimation errors step-like drop below 0.1 bin but this is still far from the target values when we have the whole ensemble of samples (Figures 9 - line a and 10 - line a). With increasing $D_{\mathrm{rs}}$ errors slowly drop but the number of iterations in the process of randomly adding additional samples relatively substantially increase (for $N=512$ we typically need 2000 iterations to achieve $\left.D_{\mathrm{rs}} \approx 0.98\right)$. Due to the randomness, some positions may be repeatedly sampled, whereas other positions are left blank.

The parametric estimation approach (Figure 9 - line c: 4parametric estimation with 9 iterations) gives better results than the non-parametric IDFT approach (Figures 9 - line b and 10

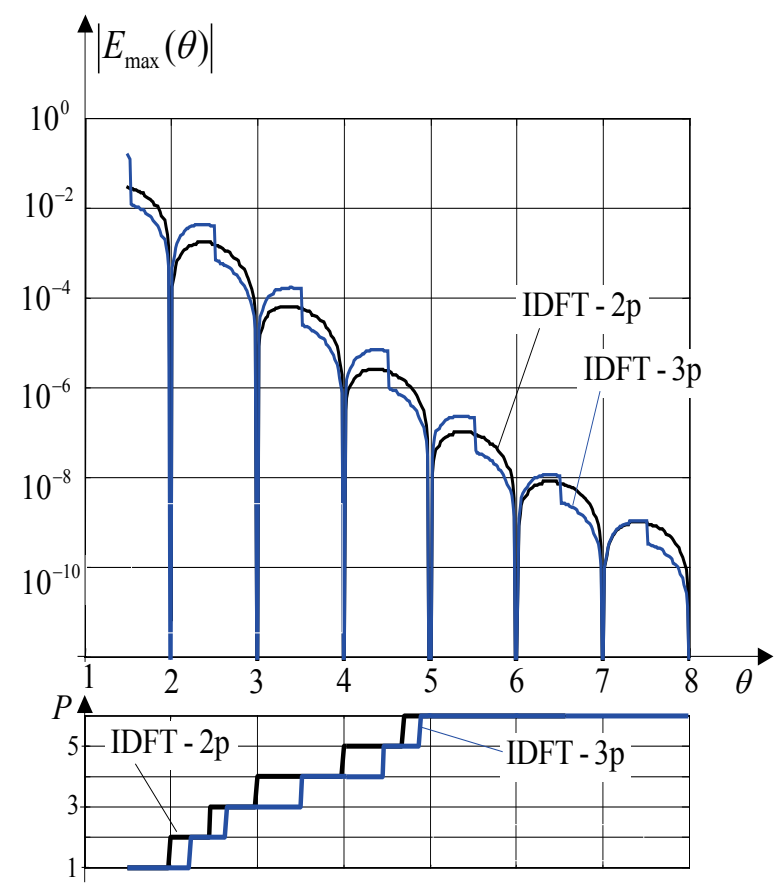

Figure 8. Minimal curves of the maximal errors of the frequency estimations with the two- (IDFT-2p) and three- (IDFT-3p) interpolations of the DFT using $\mathrm{RV}-\mathrm{I}$ windows and the corresponding orders $P=1 \ldots 6$.

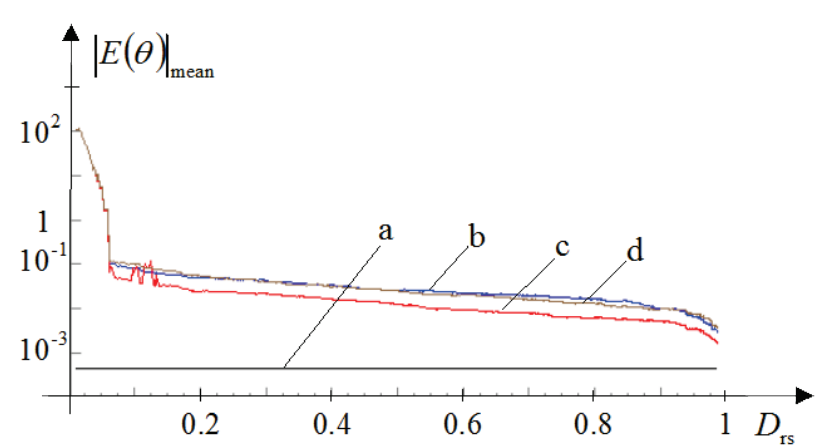

Figure 9. Mean values of the absolute errors of the frequency estimation; a - original signal - IDFT(f-3p), b - random sampled signal - IDFT(f-3p), c random sampled signal - 4-par. sine-fit (start with estimated $\mathrm{f}-3 \mathrm{p}$ ), $\mathrm{d}-$ random sampled signal - IDFT(f-2p); $\theta_{\mathrm{S}}=1, \theta=3.8, A=1, \varphi=0 \mathrm{deg}$, $N_{\text {repeat }}=100$ trials.

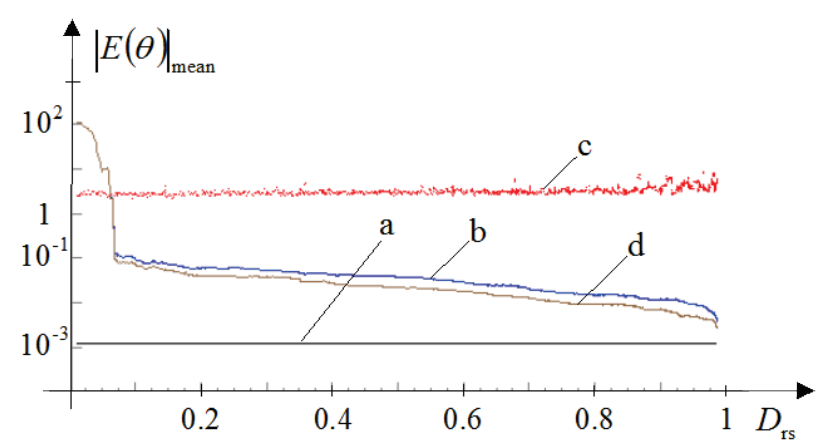

Figure 10. Mean values of the absolute errors of the frequency estimation; a - original signal - IDFT(f-3p), b - random sampled signal - IDFT(f-3p), c random sampled signal - 4-par. sine-fit (start with $\theta=2$ ), d - random sampled signal - IDFT(f-2p); $N=512, \theta_{\mathrm{S}}=1, \theta=4.4, A=1, \varphi=0 \mathrm{deg}$, $N_{\text {repeat }}=100$ trials.

line d) but the frequency has to be estimated first (typically by symmetric 3-point estimation using the Hann window $P=1$ and (2)). If we don't have a good initial value of the component frequency the parametric approach gives useless results (Figure 10 - line c).

After the point where the frequency component exceeds the random sampling noise (Figure 6 - line b: $D_{\mathrm{rs}} \doteq 0.074$ ) twopoint IDFT estimation (Figures 9 - line d and 10 - line d) gives lower errors than the three-point estimation and especially in the case of large non-coherency (Figure 10 - line d: $\delta_{m}=0.4$ ).

If the sampling frequency relatively increases to the signal frequency as in the case in Figures 1 and 2 ( $\theta_{\mathrm{S}} / \theta_{\mathrm{m}}=19.7 / 3.8>2$ ) the 'random noise' effect around the component diminishes and the non-parametric IDFT approach gives better results at lower values of $D_{\mathrm{rs}}$ (Figure 11: lines b, d).

The same behavior of the estimation errors can be noticed also for the amplitude estimations (Figures 12 and 14: $|e(A)|=\left|A_{\text {est. }} / A_{\text {true }}-1\right|$ ) and phase estimations (Figure 13: $\left.|E(\varphi)|=\left|\varphi_{\text {est. }}-\varphi_{\text {true }}\right|\right)$ where the same test conditions were used as in Figures 9 and 10. Errors drop very fast where $D_{\mathrm{rs}}$ starts increasing and after the point around $D_{\mathrm{rs}} \approx 0.1$ the decrease of the errors slowed down. 


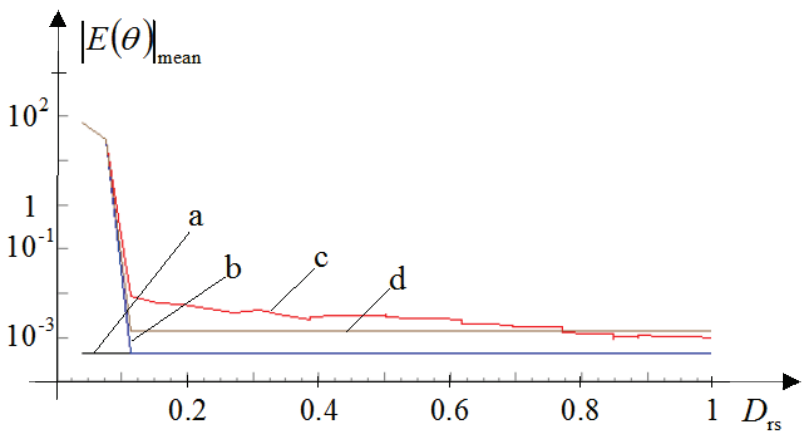

Figure 11. Mean values of the absolute errors of the frequency estimation; a - original signal - IDFT(f-3p), b - random sampled signal - IDFT(f-3p), c random sampled signal - 4-par. sine-fit (start with estimated $f-3 p$ ), d random sampled signal - IDFT(f-2p); $N=512, \theta_{\mathrm{S}}=19.7, \theta=3.8, A=1$, $\varphi=0 \mathrm{deg}, N_{\text {repeat }}=100$ trials.

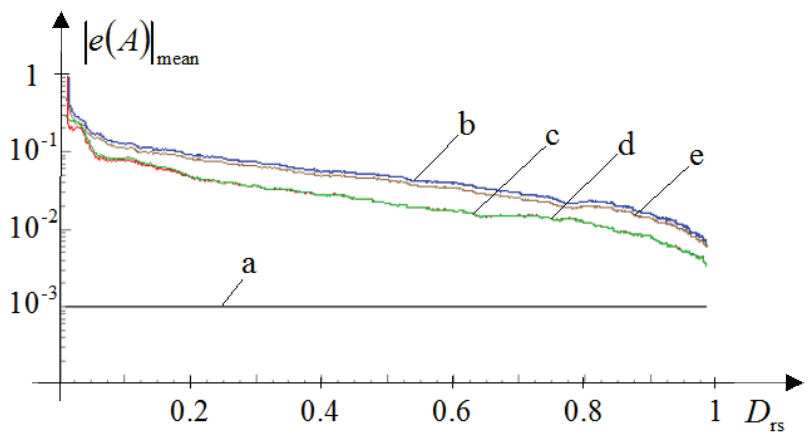

Figure 12. Mean values of the relative errors of the amplitude estimation; a - original signal - IDFT(f-3p, A-3p), b - random sampled signal - IDFT(f-3p, A-3p), c - random sampled signal - 4-par. sine-fit (start with estimated f$3 p), d$ - random sampled signal - 3-par. sine-fit (f-3p), e - random sampled signal - IDFT(f-2p, A-1p) ; $N=512, \theta_{\mathrm{S}}=1, \theta=3.8, A=1, \varphi=0 \mathrm{deg}$, $N_{\text {repeat }}=100$ trials.

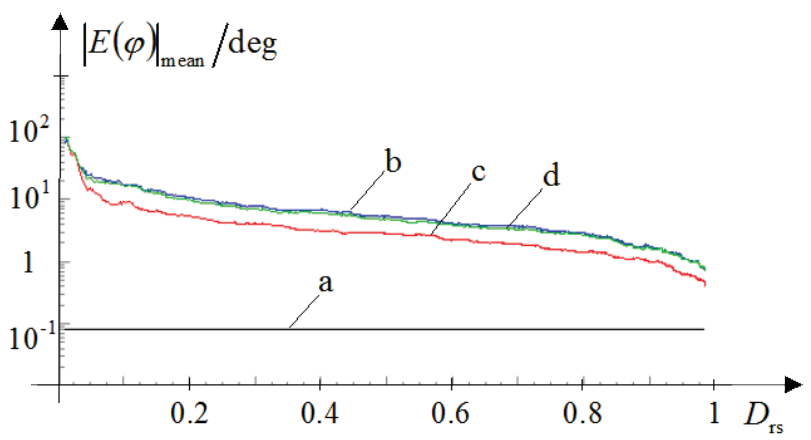

Figure 13. Mean values of the absolute errors of the phase estimation; a original signal - IDFT(f-3p, ph-3p), b - random sampled signal - IDFT(f-3p, ph-3p), c - random sampled signal - 4-par. sine-fit (start with estimated f$3 \mathrm{p}), \mathrm{d}$ - random sampled signal - 3-par. sine-fit (f-3p); $N=512, \theta_{\mathrm{S}}=1$, $\theta=3.8, A=1, N_{\text {repeat }}=100$ trials.

The iterative 4-parametric estimation (Figures 12 - line c and 13 - line c) gives a better result than one-step 3-parametric estimation (Figure 13 - line d) when the sampling frequency is relatively low but the initial frequency has to be very well estimated. If we fix the value of the estimated frequency by IDFT the one step 3-parametric sine-fit estimation gives the error values as with the non-parametric approach (Figure 13 line d).

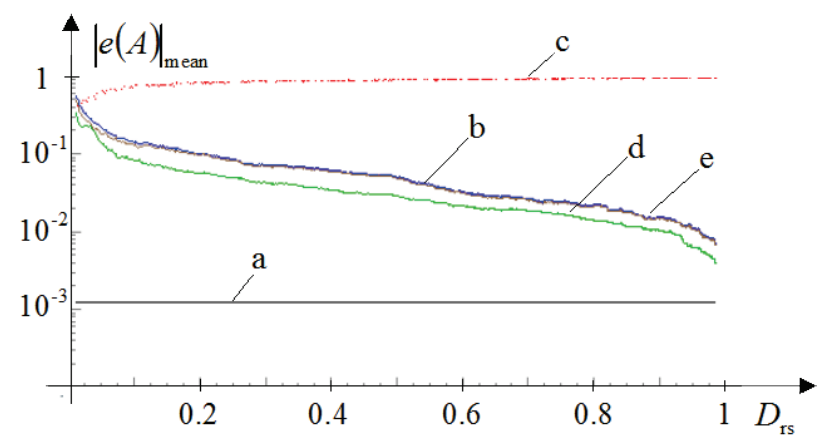

Figure 14. Mean values of the relative errors of the amplitude estimation; a - original signal - IDFT(f-3p, A-3p), b - random sampled signal - IDFT(f-3p A-3p), c - random sampled signal - 4-par. sine-fit (start with $\theta=2$ ), d random sampled signal - 3-par. sine-fit (f-3p), e - random sampled signal IDFT(f-2p, A-1p); $\quad N=512, \quad \theta_{\mathrm{S}}=1, \quad \theta=4.4, \quad A=1, \quad \varphi=0 \mathrm{deg}$, $N_{\text {repeat }}=100$ trials.

\section{EXPERIMENTAL RESULTS}

To demonstrate the behavior of estimations of the periodic signal parameters in the case of random sampling and under non-coherent sampling condition in a real measurement environment a digitizing oscilloscope (DO), Agilent 54501A [19] was used to randomly acquire signals. The signal was generated by a stable voltage generator, Keysight 33500B [20], with a nominal sine voltage of amplitude $A_{\mathrm{v}}=0.900 \mathrm{~V}$.

First, the signal frequency was fixed to $f_{\mathrm{v}}=1.9 \mathrm{MHz}$ and with time constant of $c_{\text {time }}=200 \mathrm{~ns} / \mathrm{div}$ and 10 divisions in the $\mathrm{x}$-axis, the DO acquires and shows 3.8 periods of the signal in the duration of $T_{\mathrm{M}}=2 \mu \mathrm{s} \quad\left(\theta=f_{\mathrm{v}} \cdot T_{\mathrm{M}}=1.9 \mathrm{MHz} \cdot 2 \mu \mathrm{s}=3.8\right)$. Since the DO display uses $N=500$ points to represent the signal in the $\mathrm{x}$-axis the equivalent sampling frequency was $f_{\mathrm{S}, \mathrm{eq}}=N / T_{\mathrm{M}}=250 \mathrm{MHz}$ what is 25 -times larger than the DO real sampling frequency $f_{\mathrm{S}}=10 \mathrm{MHz}$ with $\theta_{\mathrm{S}}=N \cdot f_{\mathrm{S}} / f_{\mathrm{S} \text {,eq }}=500 / 25=20$. Sampling points were acquired with the multiple 'randomly triggered' sweeps to fill the points for display presentation [19]. To fulfil all $N=500$ points typically 150 sweeps were needed and this acquisition procedure was controlled remotely by SCPI commands: :TIM:MODE SING and :RUN [19].

Figures 15 (frequency estimation) and 16 (amplitude

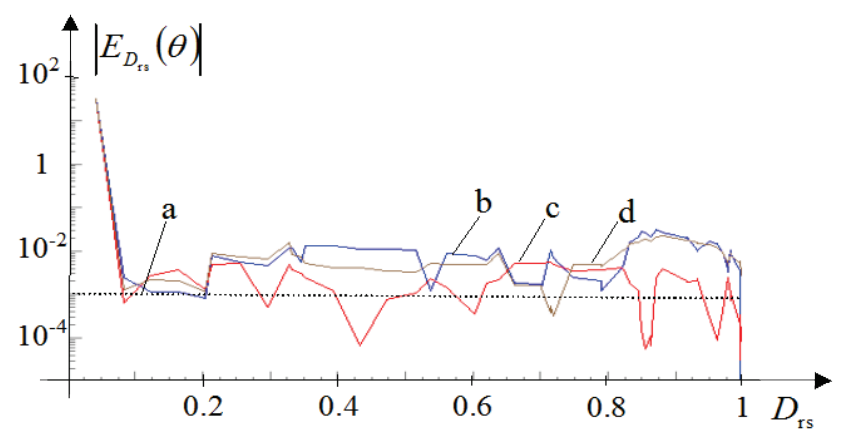

Figure 15. Mean values of the maximal absolute errors of the frequency estimation; $a$ - target estimation error, $b$ - random sampled signal - IDFT(f$3 p), c$ - random sampled signal - 4-par. sine-fit (start with estimated f-3p), d - random sampled signal - IDFT(f-2p); $N=500, \theta_{\mathrm{S}}=20, \theta=3.8$, $\varphi=0 \mathrm{deg}, N_{\text {sweeps }}=150$. 


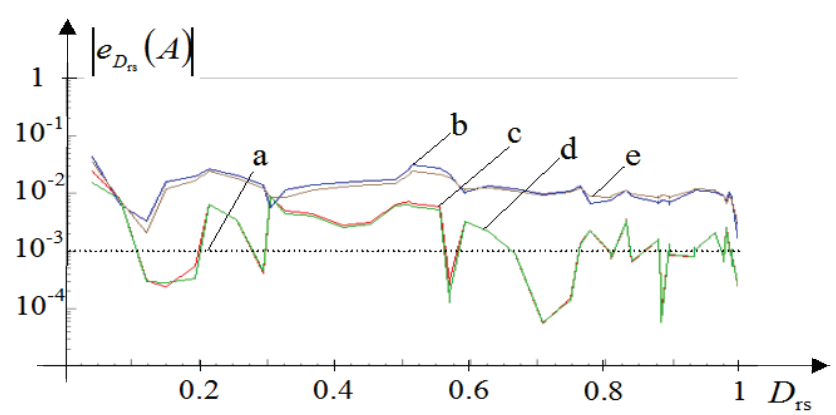

Figure 16. Mean values of the maximal relative errors of the amplitude estimation; $a$ - target estimation error, $b$ - random sampled signal - IDFT(f$3 p, A-3 p$ ), c - random sampled signal - 4-par. sine-fit (start with estimated $f-3 p), d$ - random sampled signal - 3-par. sine-fit (f-3p), e - random sampled signal - IDFT(f-2p, A-1p); $N=500, \theta_{\mathrm{S}}=20, \theta=3.8, \varphi \approx 0 \mathrm{deg}$, $N_{\text {sweeps }}=150$.

estimation) show estimation errors. The reference value for the specific parameter is the one attained with estimation on the whole number of the filled points $D_{\mathrm{rs}}=1$ after 150 sweeps with 3-point estimations ((2) and (4)).

Estimation errors are defined as $\left|E_{D_{\mathrm{rs}}}(\theta)\right|=\left|\theta_{\mathrm{est}, D_{\mathrm{rs}}}-\theta_{3 \mathrm{p}, D_{\mathrm{rs}}=1}\right|$ for the relative frequency (Figure 15) and as $\left|e_{D_{\mathrm{rs}}}(A)\right|=\left|A_{\mathrm{est}, D_{\mathrm{rs}}} / A_{3 \mathrm{p}, D_{\mathrm{rs}}=1}-1\right|$ for the amplitude (Figure 16).

Estimation error behaviors in Figure 15 confirm simulation results from Figures 9 and 11 with a very fast decrease at low values of $D_{\mathrm{rs}}<0.1$ to the target level (Figure 15 - line a: $\left|E_{D_{\mathrm{rs}}}(\theta)\right|=10^{-3}$ ) and after increasing $D_{\mathrm{rs}}>0.1$ errors drop slowly to the final estimation result with the whole ensemble of points at $D_{\mathrm{rs}}=1$.

The same estimation error behaviors can be noticed also in the case of the amplitude estimation (Figure 16). The best results are obtained by parametric sine-fit estimation procedures where the initial frequency was estimated first by the 3-point interpolated DFT approach (2).

To analyze the estimation error behavior when the relative frequency is changing the testing conditions from Figure 15 were enhanced from one frequency of $\theta=3.8$ to a frequency range $\theta=1.4 \div 6$ with frequency increments $\Delta \theta=0.05$ (Figure 17).

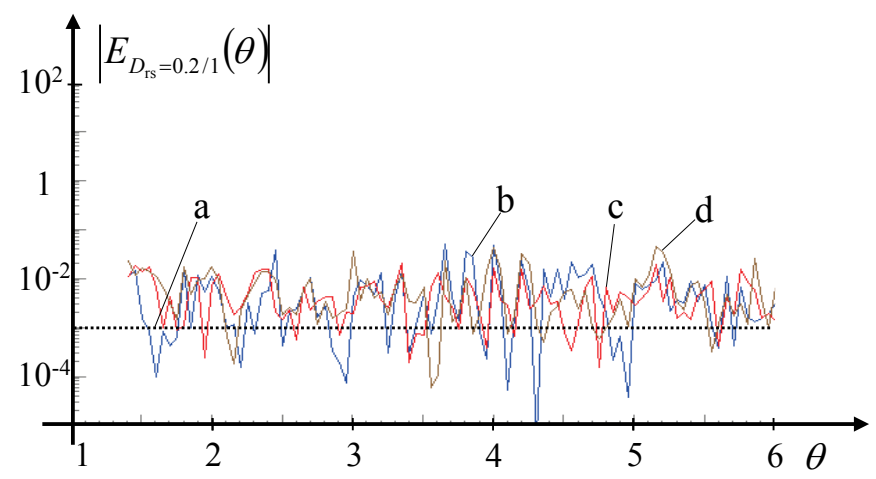

Figure 17. Mean values of the maximal absolute errors of the frequency estimation; $a$ - target estimation error, $b$ - random sampled signal - IDFT(f$3 p), c-$ random sampled signal - 4-par. sine-fit (start with estimated $f-3 p), d$ - random sampled signal-IDFT(f-2p); $N=500, \varphi \equiv 0 \mathrm{deg}, N_{\text {sweeps }}=150$.
For this testing the signal frequency was changed from $f_{\mathrm{v}, \min }=0.7 \mathrm{MHz}$ to $f_{\mathrm{v}, \text { max }}=3.0 \mathrm{MHz}$ with steps of $\Delta f_{\mathrm{v}}=25 \mathrm{kHz}$ and at each frequency the estimation error was defined as difference between estimation value around $D_{\mathrm{rs}}=0.2$ and estimation value when $D_{\mathrm{rs}}=1$ (Figure 17: $\left.\left|E_{D_{\mathrm{rs}}=0.2 / 1}(\theta)\right|=\left|\theta_{\text {est }, D_{\mathrm{rs}}=0.2}-\theta_{\text {est }, D_{\mathrm{rs}}=1}\right|\right)$. The frequency estimation errors in Figure 17 show that by changing the relative frequency the relation of errors between estimations with low sampling duty cycle and estimations with the whole ensemble of points remain on the same level.

\section{CONCLUSIONS}

Estimations of the signal component parameters from random equivalent and non-coherent sampling periodic signals comprise additional problems of random sampling noise. The non-coherency is more problematic due to frequency leakage than in the case of the coherent sampling where the problem of frequency estimation is much reduced like in approaches of compressive sensing. To achieve the lowest estimation errors the non-parametric IDFT estimation of frequency has to be used first for detection of the component frequency in both the parametric and non-parametric approach and after that the iterative parametric method should be used for other parameters when the sampling frequency is relatively lower than the Nyquist frequency and the sampling procedure becomes pure random. Simulations and experimental results show that the estimations of the component frequency, amplitude and phase are possible when the duty ratio of the random samples from the total number of samples in the non-coherent measurement interval is very low - around 0.1 .

\section{REFERENCES}

[1] Y.-C. Jenq, Perfect reconstruction of digital spectrum from nonuniformly sampled signals, IEEE Trans. Instrum. Meas. 46(3) (1997) pp. 649-652.

[2] Y. Zhao, Y. H. Hu, H. Wang, Enhanced random equivalent sampling based on compressed sensing, IEEE Trans. Instrum. Meas. 61(6) (2012) pp. 579-586.

[3] EJ. Candes, 1. Romberg, T. Tao, Robust uncertainty principles: exact signal reconstruction from highly incomplete frequency information, IEEE Trans. Inf. Theory. 52(2) (2006) pp. $489-$ 509.

[4] C. Luo, J. H. McClellan, "Discrete random sampling theory." In Proc. of ICASSP/2013 (2013) pp. 5430 - 5434.

[5] V. G. Ivchenko, A. N. Kalashnikov, R.E. Challis, B.Haves-Gill, High-speed digitizing of repetitive waveforms using accurate interleaved sampling, IEEE Trans. Instrum. Meas. 56(4) (2007) pp. 1322-1328.

[6] X. Chen at all, A sub-nyquist rate compressive sensing data acquisition front-end, IEEE J. Emerg. Select. Topics in Circ. Syst. 2(3) (2012) pp. 542-551.

[7] P. D. Hale, C. M.Wang, D. F.Williams, K. A. Remley, J.Wepman, Compensation of random and systematic timing errors in sampling oscilloscopes, IEEE Trans. Instrum. Meas. 55(6) (2006) pp. 2146-2154.

[8] P. Daponte, L. De Vito, S. Rapuano, An extension to IEEE Std. 1241 sine fit for analog-to-information converters testing, In Proc. of I²MTC/2015 Pisa, Italy (2015) pp. 1933-1937.

[9] M. Bertocco, G. Frigo, C. Narduzzi, High-accuracy frequency estimation in compressive sensing-plus-DFT spectral analysis, In Proc. of I²MTC/2015. Pisa, Italy (2015) pp. 1668-1671.

[10] D. Bao, P. Daponte, L. DE Vito, S. Rapuano, Frequencydomain characterization of random demodulation analog-to- 
information converters, ACTA IMEKO. 4(1) (2015) pp. 111120.

[11] D. Agrež, Periodic signal parameters' estimations from random sampling measurements under non-coherent sampling condition, in Proc. of the 21st Symposium IMEKO TC-4, Budapest, Hungary (2016) pp. 190-195.

[12] E.J. Candes, M.B. Wakin, An introduction to compressive sampling. IEEE Signal Proc. Magazine 25 (2008) pp. 21-30.

[13] R. Baraniuk, M. A. Davenport, M. F. Duarte, C. Hegde, J. Laska, M. Sheikh, W. Yin, An Introduction to Compressive Sensing, Rice University, Houston, Texas (2013).

[14] D. C. Rife, R. R. Boorstyn, Multiple tone parameter estimation from discrete-time observations, Bell Syst. Tech. J. 55 (1976) pp. 1389-1410.
[15] V. H. Jain, W. L. Collins, D. C. Davis, High accuracy analog measurements via interpolated FFT, IEEE Trans. Instrum. Meas. 28(1) (1979) pp. 113-122.

[16] J. Štremfelj, D. Agrež, Nonparametric estimation of power quantities in the frequency domain using Rife-Vincent windows, IEEE Trans. Instrum. Meas. 62(8) (2013) pp. 2171-2184.

[17] P. Stoica, R. Moses, Spectral analysis of signals, Prentice-Hall, NJ, USA (2005).

[18] Standard IEEE-1241-2010, IEEE Standard for Terminology and Test Methods for Analog-to-Digital Converters (2011).

[19] Agilent Technologies, 54501A Digitizing Osciloscope Programming Reference (1989).

[20] Keysight Technologies, 33500B Series Waveform Generators Data Sheet, 5991-0692EN (2015). 